\title{
From Theory to Experiment: Transformer-Based Generation Enables Rapid Discovery of Novel Reactions
}

\author{
Xinqiao Wang, ${ }^{[a] t}$ Chuansheng Yao, ${ }^{[b, c]+}$ Yun Zhang, ${ }^{[a] t}$ Jiahui Yu, ${ }^{[a]}$ Haoran Qiao, ${ }^{\left[{ }^{[d]}\right.}$ Chengyun Zhang, ${ }^{[a]}$ \\ Yejian $\mathrm{Wu}^{\left[{ }^{[a]}\right.}{ }^{\text {Renren Bai }{ }^{*}[\mathrm{~b}, \mathrm{c}]}$ and Hongliang Duan*[b,c]
}

These authors contributed equally: Xinqiao Wang, Chuansheng Yao and Yun Zhang

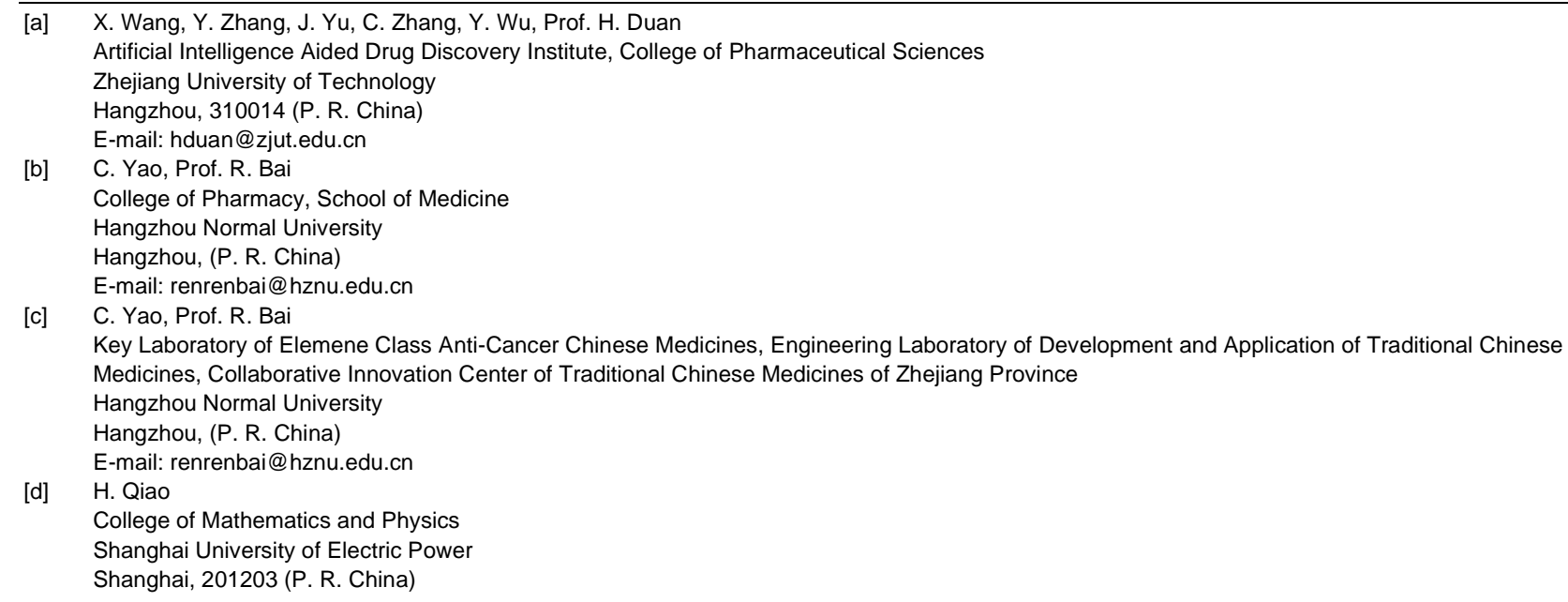

Supporting information of this article can be found under:

\begin{abstract}
Deep learning methods have been proven their potential roles in the chemical field, such as reaction prediction and retrosynthesis analysis. However, the de novo generation of unreported reactions using artificial intelligence technology remains not be completely explored. Inspired by molecular generation, we proposed the task of novel reaction generation. In this work, we applied the Heck reactions to train the transformer model, state-of-art natural language process model and obtained 4717 generated reactions after sampling and processing. We then confirmed that 2253 novel Heck reactions by organizing chemists to judge the generated reactions, and adopted organic synthesis experiment to verify the feasibility of unreported reactions. In this process, it only took 15 days from Heck reaction generation to experimental verification, proving that our model learns reaction rules in-depth and can make great contributions in the novel reaction discovery.
\end{abstract}

\section{Introduction}

The detection of new organic reactions has always been a significant research direction in the synthetic chemistry field. New reactions provide more diversiform chemical synthetic options for scientists. The discovery of the majority of new reactions was as yet guided by the "chemical intuition" of chemists, which was a time-consuming task requiring extensive experience and charactered by trial and error. For instance, it took three decades to thoroughly study the mechanism after the discovery of the
Meisenheimer rearrangement reaction by Jacob Meisenheimer in $1919 .{ }^{[1,2]}$ The long and tortuous process of discovering new reactions not only limits the development of organic synthesis methodology but also restricts the advancement of drug discovery. In recent years, several successful applications of artificial intelligence $(\mathrm{Al})$ technology in the chemistry field have offered multiple solutions to the diverse challenges of chemists. ${ }^{[3-5]}$ Scientists have explored chemical reactions from several fields, ${ }^{[6]}$ including reaction prediction and retrosynthesis analysis. ${ }^{[7-10]}$ Among others, reaction prediction, focusing on learning the rules of chemical reactions and then predicting the products with related properties from given reactants or reagents, has become an important topic in the past few years. The exists two mainstream models for reaction prediction are graph convolutional neural networks and simplified molecular-input lineentry system (SMILES)-based sequence-to-sequence (seq2seq) models. ${ }^{[11-14]}$ The transformer-based molecular transformer, proposed by Schwaller et al., is the state-of-art SMILES-based sequence-to-sequence model achieving a $90 \%$ accuracy. ${ }^{[15]}$ On the contrary, retrosynthesis analysis algorithms assist chemists in providing synthetic recommendations by outputting one step at a time or complete synthesis routes according to the given target substances. The models that learn (or infer) how to generate recommendations or use expert encoded rules or heuristics to generate recommendations are two major models of retrosynthetic planning. ${ }^{[6]}$ Such as Liu et al. applied seq2seq to retrosynthesis analysis and found that it was comparable to the rule-based expert system. ${ }^{[16]}$ However, both forward-reaction 


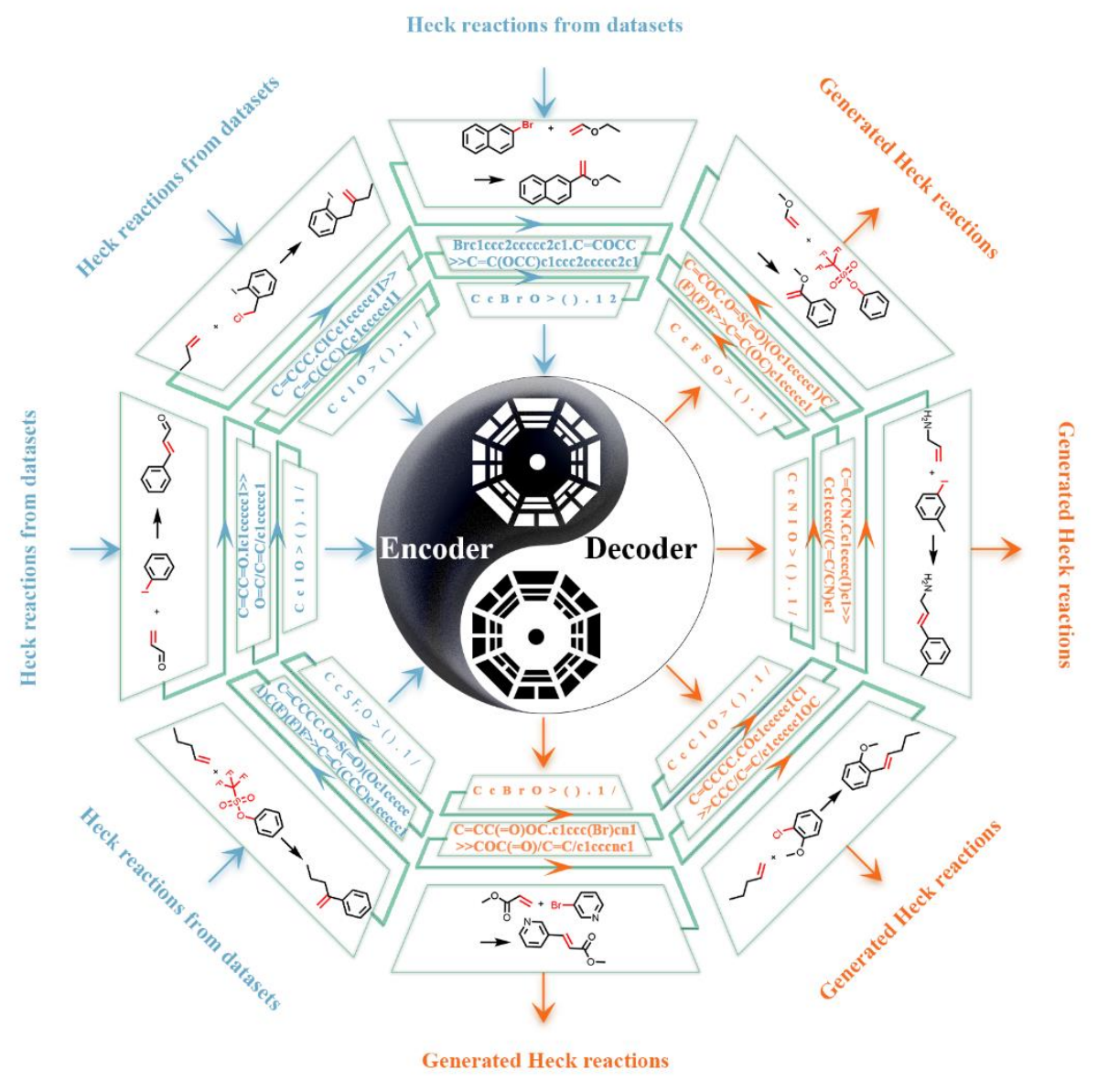

Figure 1. Schematic of the process to generate the Heck coupling reaction. Heck reactions are imported into the encoder of the model after they're converted from the 2-dimensional molecular graph to the 1-dimensional SMILES strings, decoded them then into novel Heck reactions from the decoder.

prediction and retrosynthesis planning are based on given a part of chemical reactions and outputted the rest of the reaction.

Is it feasible for deep learning models to generate a whole novel unreported reaction from scratch that is similar to the given reactions? We put forward this question inspired by de novo molecular generation, ${ }^{[17,18]}$ aiming to expand the molecular library and expedite molecular screening. Molecular generation refers to generating the active or target molecules by the chemical knowledge that the deep learning model learns from the training datasets containing numerous molecules. ${ }^{[19,20]}$ Generating molecules has been applied to various deep learning models. It is worth noting that sequence-to-sequence models have a pivotal position in the molecular generation with SMILES strings represented because of their excellent performance in text generation, such as poems generation. ${ }^{[21]}$ In particular, the recurrent neural networks (RNNs) model Segler et al. used and the generative chemical transformer model Kim et al. applied can generate a large number of high-quality molecules. ${ }^{[22,23]}$ Therefore, we attempted to apply the sequence-to-sequence model to generate new reactions like generating molecules and named it "reaction generation". The successful application of reaction generation will contribute to solve the current dilemmas of chemical reactions and accelerate the development of chemistry.

Although Bort et al. have applied recurrent neural networks and condensed graph of reaction approaches to explore the generation of Suzuki reaction, ${ }^{[24]}$ the assessment of the executive experiment was still lacked. Unlike the recurrent neural networks they used, we introduce a more powerful sequence-to-sequence model, the transformer model, that is only trained with SMILES as input. The transformer model proposed by google company for solving the machine translation task has been one of the frequently utilized neural networks in a couple of years which adopts encoder-decoder framework. ${ }^{[25]}$ Compared to previous work such as RNNs or Long Short-Term Memory (LSTM), the novelty of this model is the application of attention mechanisms in the process of sequence. In recent years, this model has gained much attention from chemistry society and completed multiple tasks in processing reactions. ${ }^{[26]}$ For instance, Vaucher et al. achieved the prediction of experimental process. ${ }^{[27]}$ Meanwhile, the transformer model has derived several forms to overcome its shortcomings such as the transformer- $\mathrm{XL}$ model. ${ }^{[28]}$ It enables learning dependency beyond a fixed-length without disrupting temporal coherence which was the problem that plagued the transformer model.

Selecting an appropriate reaction is conducive to carry out the target task of reaction generation. In this research, we selected the Heck reaction, a classic carbon-carbon coupling reaction, as a representative experiment to bring more convenience to drug discovery for its widespread application in the synthesis of alkenes. More importantly, its discoverer, R. F. Heck was awarded the Nobel Prize for its enormous contribution. ${ }^{[29]}$ And we give the mechanism in Figure S1. 


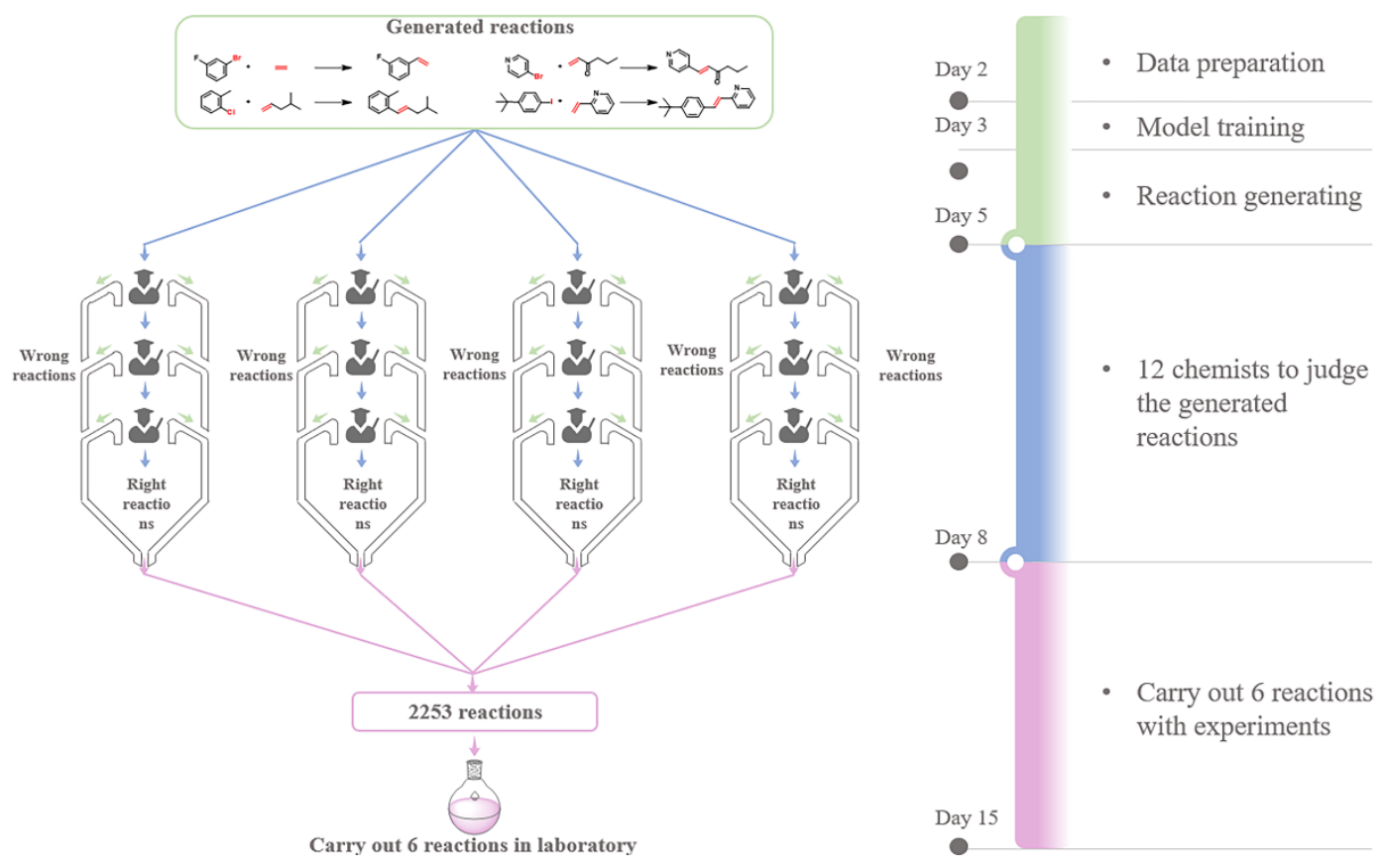

Figure 2. The flowchart of reaction generation and verification.

In this paper, we aimed at utilizing the transformer-XL model to generate Heck reaction. We constructed a training dataset of Heck coupling reaction, a dataset that is downloaded from "Reaxys" database and imported into the model to generate the new Heck reactions (see Figure 1). Even more importantly, we organized 12 academic chemists to analyze and assess the thousands of generative reactions according to chemical experience and integrating them with the "Scifinder" and "Reaxys" database. Since some of the reactions generated by our model are completely new and have not been reported in the literature, we performed actual organic chemical synthesis experiments to verify whether the reactions generated by the model are feasible and whether the configuration of the compounds given by the model is correct. Furthermore, in accordance with the availability of raw materials, we chose 8 of the generative unreported reactions to confirm the chemical feasibility. With the Heck reaction are successfully generated and supported by experiments, it not only provides us a handier way for detection of novel reactions but a means of verification for the chemical-level generation model.

\section{Results and Discussion}

We totally spent 15 days to explore novel Heck reactions with the transformer- $\mathrm{XL}$ model (Figure 2). We first took 2 days to prepare the data (see Method Dataset), and then imported the training dataset comprised of 8863 Heck reactions to the model. Finally, after 2 days of generating and then removing all duplicates, we obtained 4717 reactions that were not included in the training and validation datasets. Then, we organized 12 experimental chemists to judge the validation of reactions. The chemists were divided into four groups and each group was responsible for a quarter of the total generated reactions. Only the reaction was simultaneously considered as feasibility by the three chemists, we retained and converged it to a dataset consisting of

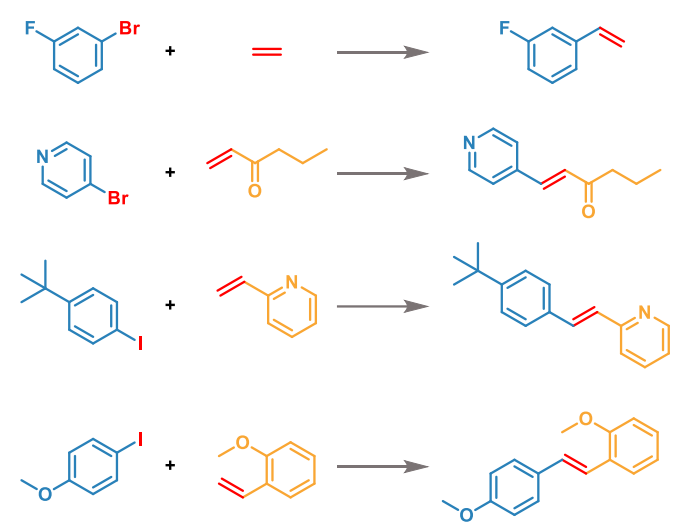

Figure 3. Examples of unreported generated Heck reactions.

2253 reactions after 3 days. A few representative examples of generated reactions are depicted in Figure 3 . Out of these reactions, they are logical with reasonable reactants and reaction centers matching Heck reactions. Further, we spent 7 days to verification in laboratory.

During the 2 days' process of generating reactions, we artificially assign it as two steps which do not exist in practical application.

(I) The generation of reactants and products molecules.

(II) The process to correspond the reactants and products. It refers to the formation of the arrows connected reactants and products in the two-dimensional diagram of reactions.

In stage I, each reaction is composed of at least one reactant and one product so the prerequisite of valid reaction is that both reactant and product are effective. Interestingly, despite that the 4717 novel reactions are not all valid reactions, all of the corresponding reactants and products are valid SMILES formula, indicating that the generative ability of molecular of the model is impeccable. Meanwhile, we applied the t-SNE (t-distributed 


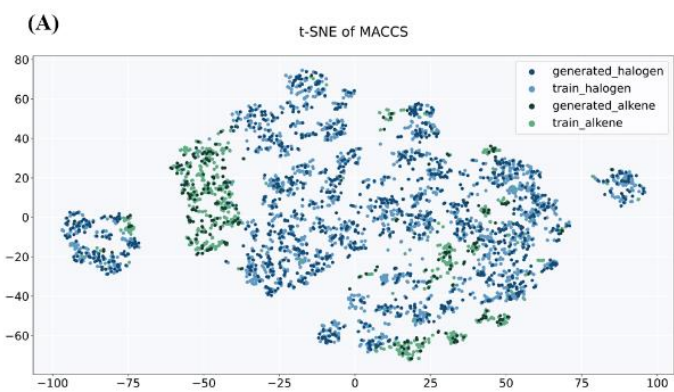

(C)

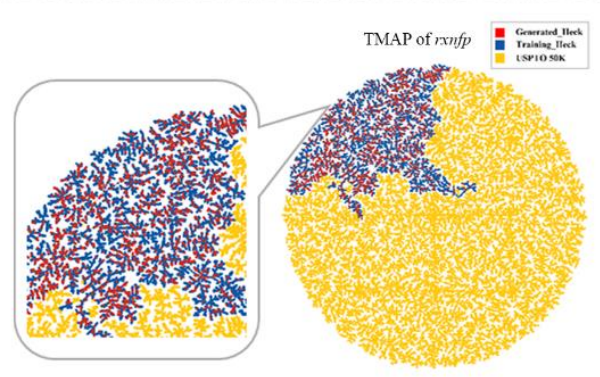

(B)

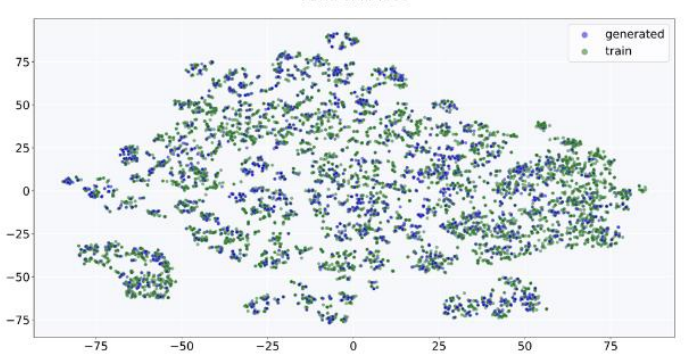

(D)

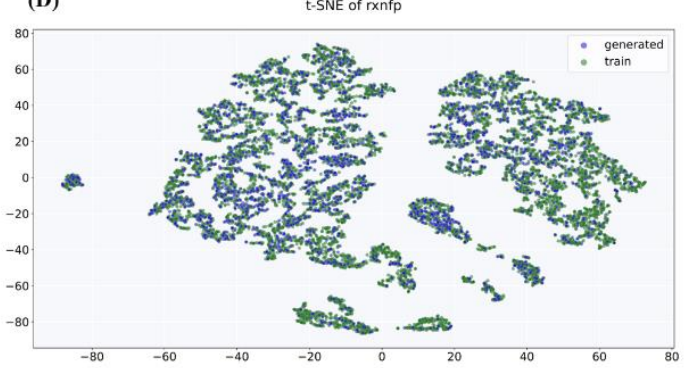

Figure 4. The plot of distribution of reactants, products and reactions. (A) The t-SNE plot of MACCS of reactants. Halogenated aromatics from training set (blue) and generated set (deep blue), and alkenes from training set (green) and generated set (deep green). (B) The t-SNE plot of products from training set (green) and generated set (blue). (C) The TMAP plot of rxnfp of reactions from training set (blue), generated set (red) and USPTO 50K (yellow). (D) The t-SNE plot of rxnfp of reactions from training set (green) and generated set (blue).

Table 1. Distribution of alkene reactants in the training set and generated set.

\begin{tabular}{ccccc}
\hline $\begin{array}{c}\text { carbon-carbon double } \\
\text { bond classification of } \\
\text { reactants }\end{array}$ & \multicolumn{2}{c}{ Amount } & \multicolumn{2}{c}{ Rate (\%) } \\
\cline { 2 - 5 } Ethylene & 141 & 9 & 1.57 & 0.40 \\
\hline Training & Generated & Training & Generated \\
Monosubstituted & 8300 & 2179 & 92.60 & 96.72 \\
Disubstituted & 502 & 64 & 5.60 & 2.84 \\
Trisubstituted & 20 & 1 & 0.23 & 0.04 \\
Total & 8963 & 2253 & 100 & 100 \\
\hline
\end{tabular}

Table 2. Distribution of halogenated aromatics and trifluoromethanesulfonate derivatives in training set and generated set.

\begin{tabular}{ccccc}
\hline \multirow{2}{*}{$\begin{array}{c}\text { Halogen atoms } \\
\text { classification of } \\
\text { reactants }\end{array}$} & \multicolumn{2}{c}{ Amount } & \multicolumn{2}{c}{ Rate (\%) } \\
\cline { 2 - 5 } Cl & 471 & 161 & 5.3 & 7.1 \\
$\mathrm{Br}$ & 4939 & 1356 & 55.1 & 60.2 \\
$\mathrm{I}$ & 3274 & 670 & 36.5 & 29.8 \\
OTf & 279 & 66 & 3.1 & 2.9 \\
Total & 8963 & 2253 & 100 & 100
\end{tabular}

stochastic neighbor embedding, a technique similar to PCA) approach to visualize MACCS fingerprints to further verify the validation of generated molecules. ${ }^{[30]}$ The t-SNE is a variation of Stochastic Neighbor Embedding (Hinton et al.) that visualizes high-dimensional data by giving each datapoint a location in a two or three-dimensional map. ${ }^{[31]}$ And MACCS is a molecular fingerprint with 166 dimensions and each dimension corresponds to a functional group, which is suitable for reactants and products' focus. Figure $4 \mathrm{~A}$ is the t-SNE plot of the MACCS fingerprints of the reactants of the generated reaction and the reactants of the training set, while the t-SNE plot of products is shown in Figure 4B. As expected, the training molecules completely overlap with the corresponding generated molecular set, which indicates that the model has generated many similar molecules around the training set.

At the same time, we classified the reactants in the training set and the 2253 reactions generation set. Table 1 showed the distribution of alkenes according to the number of substituents around carbon-carbon double bond, while Table 2 is the classification of halogenated aromatic hydrocarbons and trifluoromethanesulfonate derivatives. It can be found that the generated monosubstituted alkenes, bromo- aromatic hydrocarbons and iodinated aromatic hydrocarbons occupy the majority of the generated reactants, which is similar to the distribution of these three reactants in the training set. Meanwhile, the generated reactions covered all alkene types, although some types of alkenes are few, it was still an indication of the integrity of molecular information, well preserved in the process of generating molecules in the stage I.

In stage II, the process of combining the corresponding reactant and product molecules into a reaction means that the model must learn the Heck reaction rules. Despite that the Heck coupling reaction is one of the most widely used catalytic carbon- 
carbon bond forming tools in organic synthesis, its rules are complicated for the transformer-XL model. To further prove that the reactions generated by the model belong to the Heck reaction, we use TMAP to visualize the rxnfp of the reactions. Schwaller et al. reported that the representations learned by the Bidirectional Encoder Representations from Transformers (BERT) can be used as reaction fingerprints ( $r x n f p)$, which were independent of the number of molecules involved in a reaction. ${ }^{[32]}$ And then mapped rxnfp to the TMAP, a method to visualize high-dimensional spaces as tree-like graphs. ${ }^{[3]}$ As shown in Figure $4 \mathrm{C}$, we connected the 2253 chemist-judged reactions in the generated dataset and the reactions in the training dataset, each represented as a point, according to the similarity measured by the rxnfp. Additionally, the $50 \mathrm{~K}$ reactions downloaded and curated by Liu et al. from the United States Patent Trademark office (referred to hereafter as USPTO 50K) are used to form the backbone of chemical space as the USPTO $50 \mathrm{~K}$ contains varieties of chemical reactions. ${ }^{[16]}$ Color-coding by the three classifications of reaction dataset above showed that the generated 2253 reactions and training set formed a good overlap, demonstrating that the 2253 reactions judged by chemists are totally Heck coupling reactions. In addition, we testified the category of reaction using t-SNE to dimensionally reduce the rxnfp of the dataset (Figure 4D). The result proved that

Table 3. Distribution of Heck reactions in training set and generated set.

\begin{tabular}{ccccc}
\hline \multirow{2}{*}{$\begin{array}{c}\text { Classification of } \\
\text { reaction type }\end{array}$} & \multicolumn{2}{c}{ Amount } & \multicolumn{2}{c}{ Rate (\%) } \\
\cline { 2 - 5 } & Training & Generated & Training & Generated \\
\hline intermolecular reaction & 8464 & 2213 & 94.4 & 98.2 \\
intramolecular reaction & 499 & 40 & 5.6 & 1.8 \\
Total & 8963 & 2253 & 100 & 100 \\
\hline
\end{tabular}
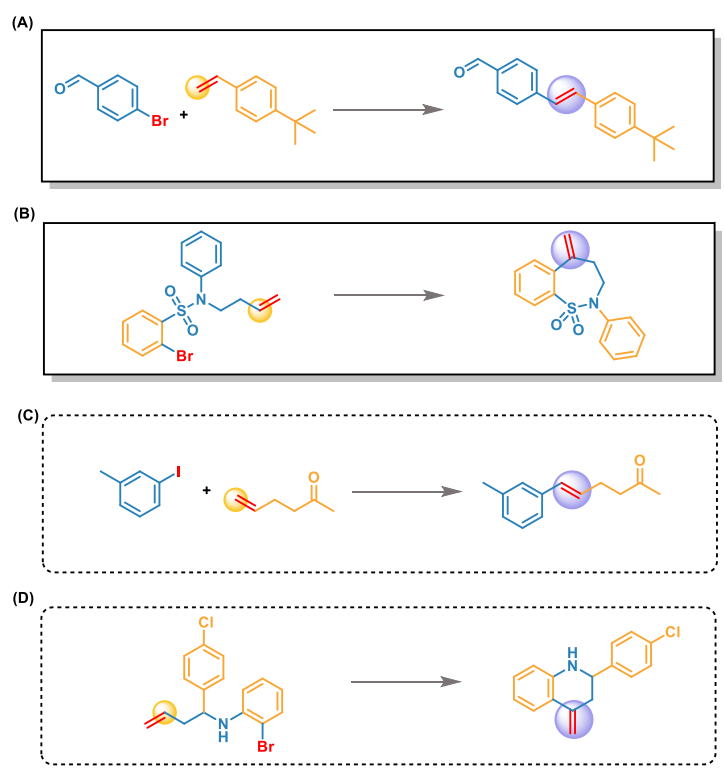

Figure 5. The representative examples of the intramolecular and intermolecular Heck reaction. (A) Intermolecular Heck reaction from the training set. (B) Intramolecular Heck reaction from the training set. (C) Intermolecular Heck reaction from the generated set. (D) Intramolecular Heck reaction from the generated set. the model had relatively well mastered the reaction rules from the training set and reproduced it.

To explore in detail whether our model fully understands the Heck reaction, we conducted an in-depth analysis of the generated Heck reaction set. First, we divided the Heck reaction into intermolecular and intramolecular reactions. Among the training dataset, there are 8464 intermolecular reactions and 499 intramolecular reactions (Table 3). As for the generated reactions, the intermolecular reaction accounts for $98.2 \%$, which is consistent with the characteristic of numerous intermolecular reactions existed in the distribution of the training dataset. In Figure 5, we listed several representative examples of intermolecular reactions and intramolecular reactions from training and generated datasets.

For the intermolecular reactions, we analyzed from the following three aspects: regioselectivity, stereoselectivity and chemoselectivity. Based on the Heck reaction mechanism, the migration insertion of alkenes is the determining step of regioselectivity, while stereoselectivity involves the elimination of $\beta$ hydrogen on carbon-carbon double bond. Therefore, we analyzed the region- and stereoselectivity of the generated reactions from the perspective of alkenes. Regioselectivity implies that there is one functional group that can react in two different sites and a reagent must choose where to react (Figure 6A). For the Heck coupling reaction, regioselectivity has always been an unavoidable problem. For reactions with ethylene as reactants, the process of occurring reactions does not involve regioselectivity since the left and right sites of alkene are equivalent for insertion. Moreover, disubstituted and trisubstituted alkenes were not worth discussing the regioselectivity. Therefore, we mainly discussed the regioselectivity of monosubstituted alkenes. Generally, the regioselectivity of monosubstituted alkenes is determined by the group attached to the double bond. As shown in Figure 6B, 4-bromopyridine reacted with hex-1-en-3one to produce 1,2-disubstituted alkenes. Because the new carbon-carbon bond forms at the other end of the alkene when the alkene is polarized by an electron-withdrawing group. The carbonyl group around the alkene of 1-hexanone happens to be an electron withdrawing group, so the reaction site located at the $\beta$ position. Due to steric hindrance, the arylation of monosubstituted alkenes is more likely to occur at the $\beta$-position. Moreover, we observe that more reaction sites are located at $\beta$ positions in the generated reactions (Table 4). In contrast, electron-donating groups lead to produce the 1,1-disubstituted product, such as ether group. As another example shown in Figure $6 \mathrm{~B}$, the model could capture the information that the oxygen located in ethers is an electron-donating group and produce a product of 1,1-disubstituted.

To further explore the stereoselectivity understanding of Heck reaction of our model, we gave an introduction to stereoselectivity in Figure $6 \mathrm{~A}$, referring to how they reacted (stereochemistry of the products). For ethylene and trisubstituted alkenes, they are not in the scope of the discussion because of without existing stereoselectivity. For monosubstituted alkenes, the stereoselectivity is partially related to regioselectivity. There is only one case of stereoselectivity for monosubstituted alkenes if the reaction site is located at the $\alpha$-position, which is similar to ethylene, the alkenes will become the terminal alkenes. However, the situation becomes complicated when the reaction site is located in the $\beta$-position. As shown in Figure $6(\mathrm{C})(\mathrm{a})$, when 1 iodo-3-methylbenzene and hex-5-en-2-one react, their product 


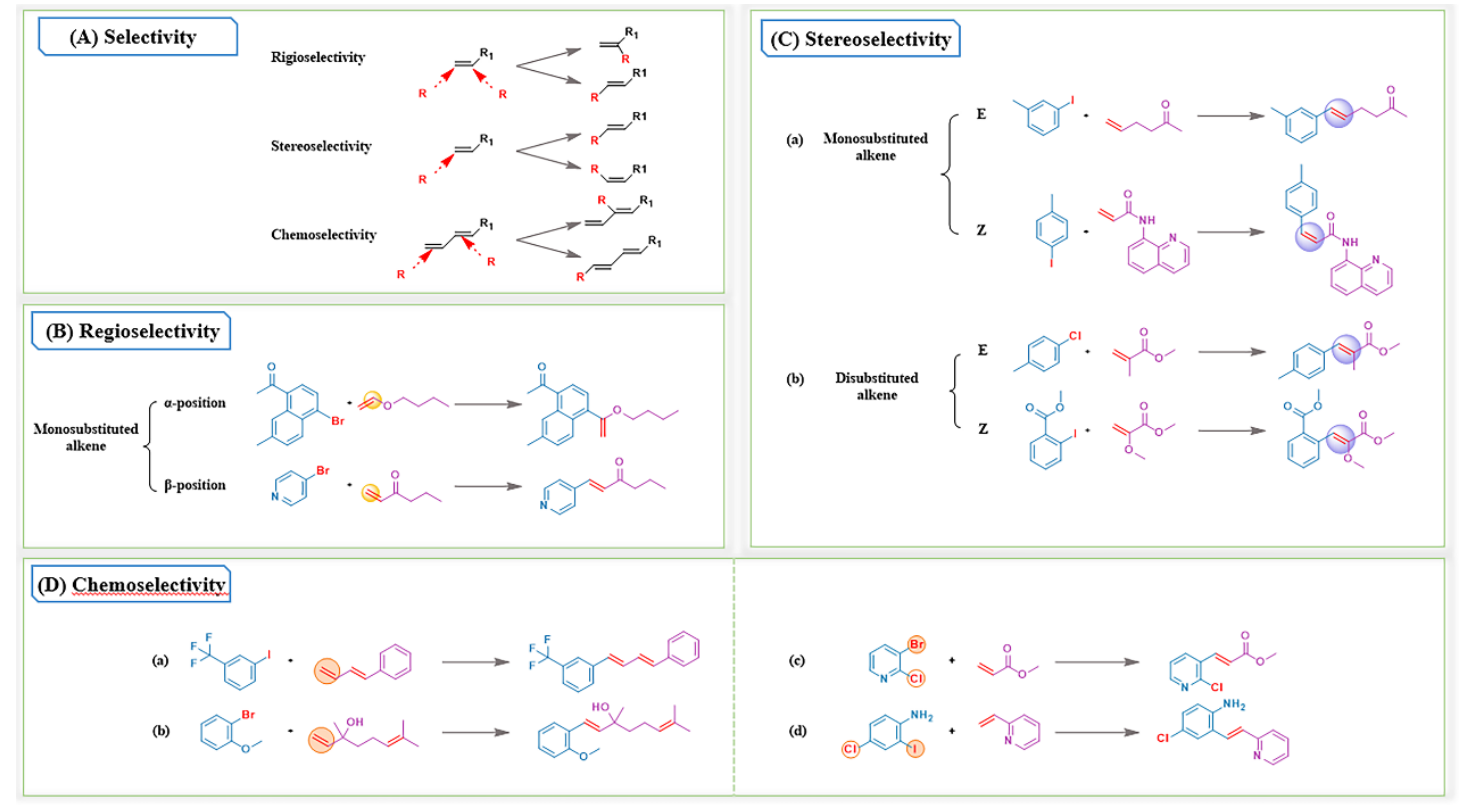

Figure 6. Analyze the generated novel reactions from the perspective of the selectivity of the Heck reaction. (A) The definition of the regio-, stereo-, chemoselectivity. (B) Analysis of the regioselectivity of alkenes. (C) Analysis of the stereoselectivity of alkenes. (D) Analysis of the chemoselectivity of alkenes.

Table 4. Regio- and stereoselectivity of monosubstituted alkenes in the training set and generated set.

\begin{tabular}{|c|c|c|c|c|c|}
\hline \multirow{2}{*}{\multicolumn{2}{|c|}{$\begin{array}{l}\text { Classification of } \\
\text { monosubstituted } \\
\text { alkenes }\end{array}$}} & \multicolumn{2}{|c|}{ Amount } & \multicolumn{2}{|c|}{ Rate (\%) } \\
\hline & & Training & Generated & Training & Generated \\
\hline \multicolumn{2}{|c|}{ a-position } & 440 & 62 & 5.59 & 2.90 \\
\hline \multirow{2}{*}{$\beta$-position } & E & 7397 & 2078 & 94.04 & 97.06 \\
\hline & Z & 29 & 1 & 0.37 & 0.04 \\
\hline \multicolumn{2}{|c|}{ Total } & 7866 & 2141 & 100 & 100 \\
\hline
\end{tabular}

will be $E$-isomer. Because the trans-alkene product will be easy to obtained which is more stable on thermodynamics, since only $\beta$-hydrogens located on the same side of the $\mathrm{Pd}$ atom can be eliminated, and the steric hindrance of the substituent around the carbon-carbon double bind. In the generated reactions. We simultaneously find that $(Z)-1,2$ disubstituted alkenes appear in the product (Figure $6 \mathrm{C}(\mathrm{a})$ ), while the most common product is $(E)$ 1,2 disubstituted alkenes. But there is a considerable amount of literature in the area of $(Z)-1,2$ disubstituted alkenes as Cheng et al. reported. ${ }^{[34]}$ For disubstituted alkenes, the elimination of $\beta$ hydrogen occurs in the process when the benzene ring is coplanar with the small substituent. In other words, the benzene ring and the large sterically hindering group are trans-coplanar when the product is generated. As illustrated in Figure $6 \mathrm{C}(\mathrm{b})$, the benzene ring is coplanar with methyl or methoxy because of the steric hindrance of methoxycarbonyl, and then generate the $E$ isomer product or the Z-isomer product. And Table 4 shows the number of reactions in all categories of the stereo configuration of monosubstituted alkenes, demonstrating that the $E$-isomer products are far more than the $Z$-isomer products, this conforms with the situation we discussed earlier.

Finally, we discussed the degree that the model learned the chemoselectivity of intermolecular reactions. Chemoselectivity re-

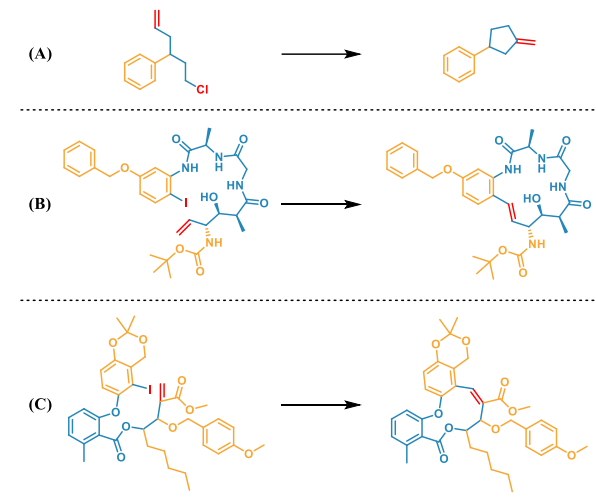

Figure 7. Examples of generated intramolecular Heck reactions.

fers to the competition between different functional groups (Figure $6 A)$. The difference is that chemoselectivity is not only related to alkenes but also deals with halogenated aromatics. We can well perceive from Figure $6 \mathrm{D}(\mathrm{a})$ and Figure $6 \mathrm{D}(\mathrm{b})$ that the benzene ring preferentially reacts with the monosubstituted alkenes when monosubstituted double bonds, disubstituted or trisubstituted double bonds, were simultaneously present in the reactants. Because the number of substituents on the carbon-carbon double bond determines the reactivity of the alkenes in the Heck reaction, and as the number of substituents increases, the reaction rate and yield will decrease. Among the four types of alkenes in our classification, the reactions with the trisubstituted alkenes generally have the slowest reaction rate and lowest yield.

Similarly, alkenes will prefer one of halogens to react when there are multiple halogens on the aromatic ring. As shown in Figure $6 \mathrm{D}(\mathrm{c})$, while bromine and chlorine are both reactive sites, the model believes that bromine will react in preference to chlorine, and the model also gave a similar conclusion that the reaction activity of iodine is greater than chlorine in Figure $6 \mathrm{D}(\mathrm{d})$. We 
Table 5. The comparison between generated reactions and experimental reactions.

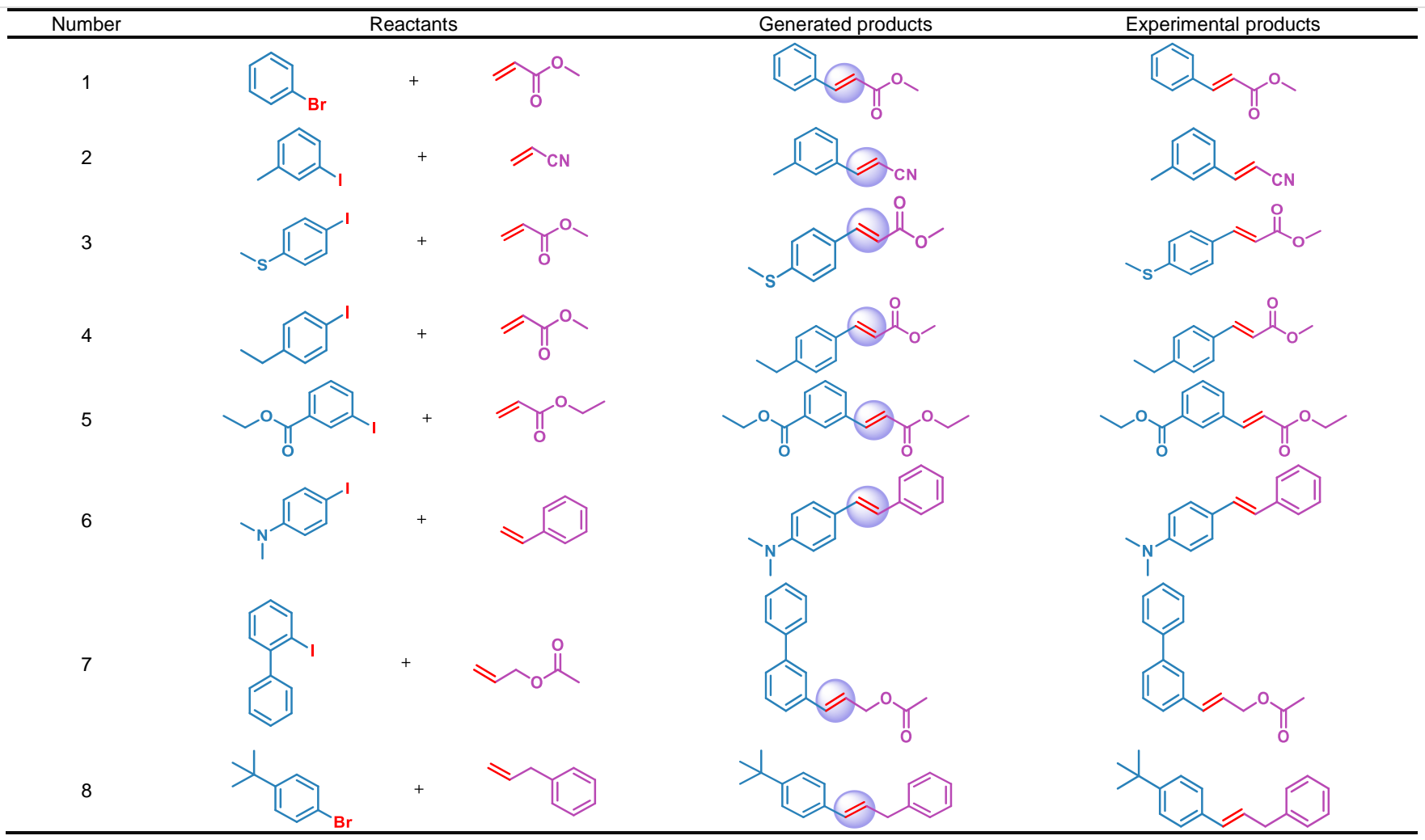

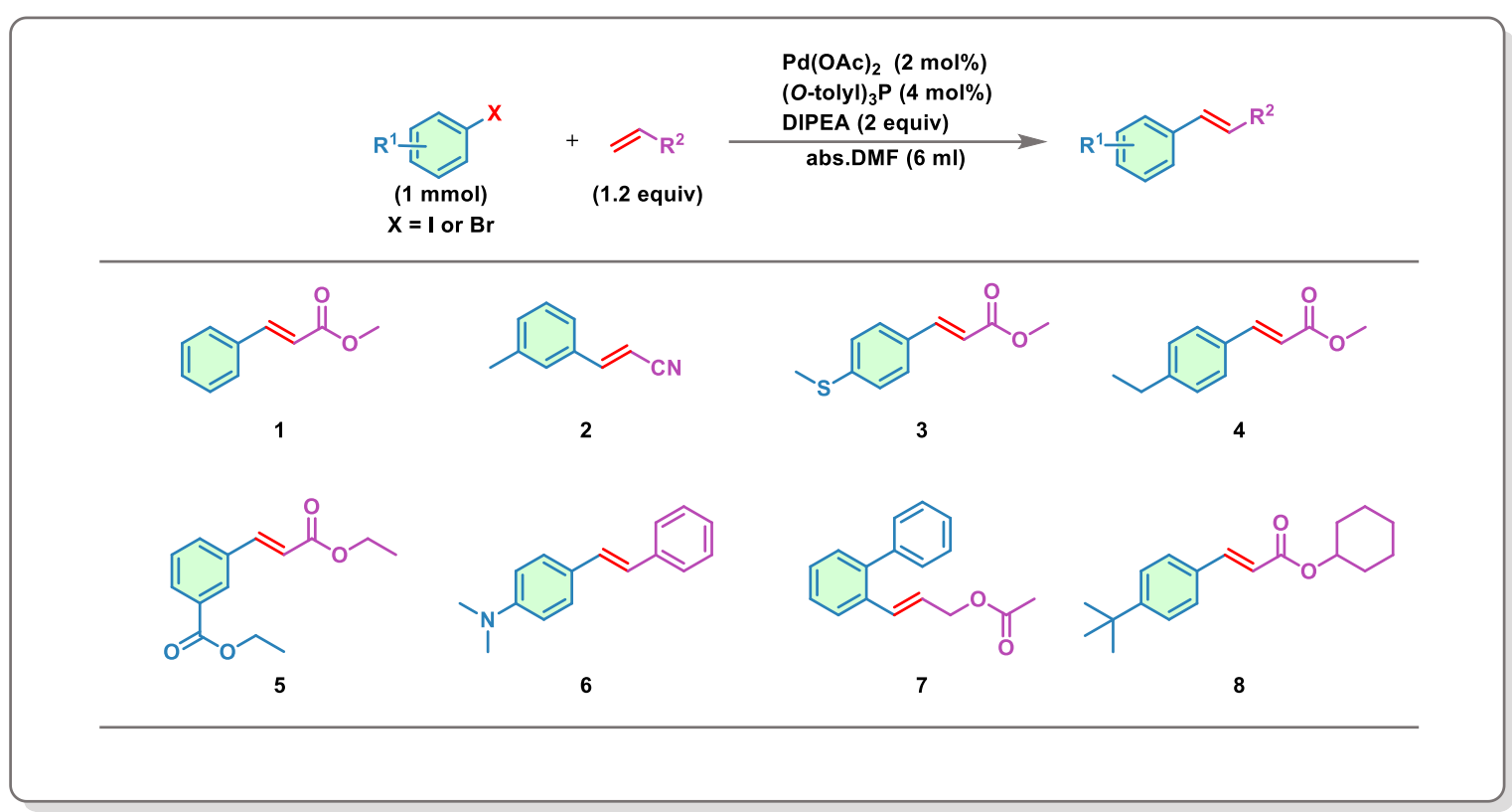

Figure 8. $\mathrm{Pd}(\mathrm{OAc})_{2}$-Catalyzed Heck reactions.

further find that the reaction priority is $\mathrm{I}>>\mathrm{OTf}>\mathrm{Br}>>\mathrm{Cl}$. This is owing to the different reaction rates of different halogenated aromatic hydrocarbons during the oxidative addition process of the Heck reaction. Among them, iodoaromatics not only have fast reaction speed and high yield, but also mild reaction conditions, so they have become the most commonly used Heck reaction substrates. The brominated aromatic hydrocarbons have also been favored by chemists due to their low price. And although trifluoromethanesulfonic acid derivatives have higher reactivity they are rarely used because of the unavailability of raw materials which will lengthen the experiment period. These also explain why the bromine and iodine reactions obviously account for the majority in both the training set and the generation set as shown in Table 2.

Within the past three decades, the intramolecular Heck reaction has emerged as a particularly versatile and reliable 
carbon-carbon bond-forming process, which allows for the construction of the whole spectrum of ring sizes: small $(n=3$ or $4)$, normal $(n=5,6$ or 7$)$, medium $(n=8-14)$ and large $(n>14)$. For the intramolecular reactions with $\beta$-hydrogens available for elimination on both sides of alkenes, the general ring formation rule is to preferentially generate relatively small cyclic compounds while the ring size is normal. ${ }^{[35]}$ For example, in the selection of generating a five-membered ring and a six-membered ring, the five-membered ring is preferentially generated (Figure 7A). The successful application of this type of reaction that generates extraring double bonds is of great significance because the exocyclic double bond is a major problem in synthesis. The intramolecular Heck reaction also allows for the synthesis of exo or endo medium-sized and even large rings, and most of the products produced are in the E-form configuration because the ring tension is relatively small in large rings. Figure 7B shows the 15 -endo cyclization products. The situation changed while there is no $\beta$ hydrogen can be eliminated on one side of the alkenes. Fig $7 \mathrm{C}$ shows that it is undoubtedly that there is only one kind of 11-endo cyclization product when there is only one position for $\mathrm{Pd}$ atom to insert.

In the analysis of intramolecular reactions and region-, stereo-, chemoselectivity of intermolecular reactions, we confirmed that all of the 2253 reactions are theoretically feasible, which proved that our model had a very sufficient understanding of the rules of chemical reactions.

We then conducted experiments to verify these feasible reactions to prove their feasibility from a practical point of view. We chose 2026 reactions with iodine or bromine as the reaction center among 2253 reactions since chlorine has low activity and trifluoromethanesulfonate derivatives are more difficult to obtain. We finally carried out 8 verification reactions based on the availability of the corresponding reactants and reagents. Table 6 lists the reactions we chose and the final products obtained in the laboratory. It shows that both the products generated by the model and the real products obtained by the experiment are in full compliance with our previous analysis by comparison. It is worth noting that most of the Heck reaction conditions are relatively uniform. So, although our generative model have the ability of generating the corresponding reaction conditions for each Heck reaction, we still chose the more common reaction conditions in the experimental verification, took $\mathrm{Pd}(\mathrm{OAc})_{2}$ as the catalyst, (Otolyl) ${ }_{3} \mathrm{P}$ as ligand, DIPEA as base(Figure 8 ). As a specific example, the generated product of reaction 1 in Table 5 are 1,2disubstituted because of the electron withdrawing group connected around the double bond, and we obtained the expected ${ }^{1} \mathrm{H}$ NMR and ${ }^{13} \mathrm{C}$ NMR spectra of products in experiment with generated reactant (FigureS3). Similarly, spectra of the other 7 products can be found in Figure S4-Figure S10. This demonstrated that the model showed the correct prediction for the regioselectivity and stereoselectivity of these reactions.

\section{Conclusion}

In this work, we trained the transformer-XL model with the dataset containing 8863 Heck reactions, and finally obtained a total of 2253 novel Heck reactions evaluated by chemists. We further analyzed whether the generated reaction learned the rules of Heck reaction by analyzing them in terms of regioselectivity, stereoselectivity, chemoselectivity, etc. Since in chemistry, a miss is as good as a mile, we performed 6 generated reactions as rigorous experimental verification and observe the consistency between the generated and experimental products. In this way, we proved the feasibility of reaction generation, and the transformer-XL model with a thorough comprehension of reactions showed the ability of helping to generate feasible and novel reactions.

Different from the other generation task applied in natural language processing (NLP), such as poetry, novels and even molecular generation, which is difficult to have a quantitative standard to measure the quality of the generated content However, in our reaction generation task, the results generated by the model have only two results, right or wrong, and there is no ambiguity. Although there is greater space for optimization of the project, it still has provided a new direction to the exploration of chemical reactions. The combination of $\mathrm{Al}$ and chemical reactions can accelerate the discovery of novel reactions and further accelerate the drug discovery.

\section{Computational Methods}

Dataset. The reaction generative model is trained on a SMILES file containing only Heck coupling reactions, which are extracted from the "Reaxys" database based on the retrieval of reaction template and/or reaction name (all entries that use the phrase "Heck reaction"). The extracted Microsoft Excel files undergo a series of postprocessing processes with python scripts to obtain a high-quality dataset meeting the requirement of generating new reactions. In this step, inadequate reactions that the SMILES string is missing corresponding to either reactant or products and that have the same reactant and product are removed from the file. And for reactions with identical reaction SMILES we retained only one copy. Finally, a dataset containing 9959 Heck reaction is connected based on Heck reaction template with a Python script utilizing the RDkit and is divided into training set and validation set (9:1).

Model. We selected the transformer- $\mathrm{XL}$ model as a generation model, which is the state-of-art method that combined artificial intelligence with the chemical field. It consists of encoder and decoder architecture, and an "attention" mechanism was added to connect the encoder and decoder. Because of entire dependent on the attention mechanism, the model avoids recurrence and draws global dependencies between input and output. In addition, every encoder and decoder structure includes several feed forward layers, in which the chemical information the transformer- $\mathrm{XL}$ model learned from the training dataset stores.

To match the algorithms of the transformer-XL models. We imported the reactions with "simple molecular-input line-entry system" (SMILES) strings. We use letters to represent atoms and numbers to represent the number of rings. For example, in Figure

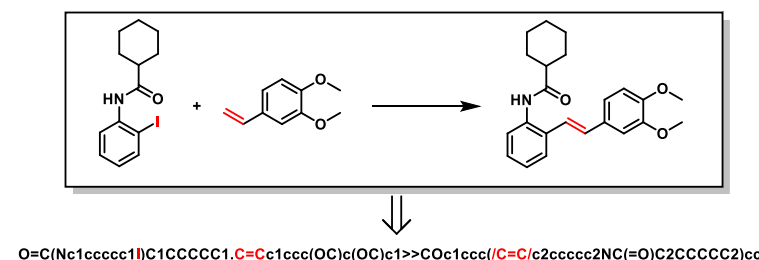

Figure 9. Mutual conversions between SMILES language and molecular structure. 


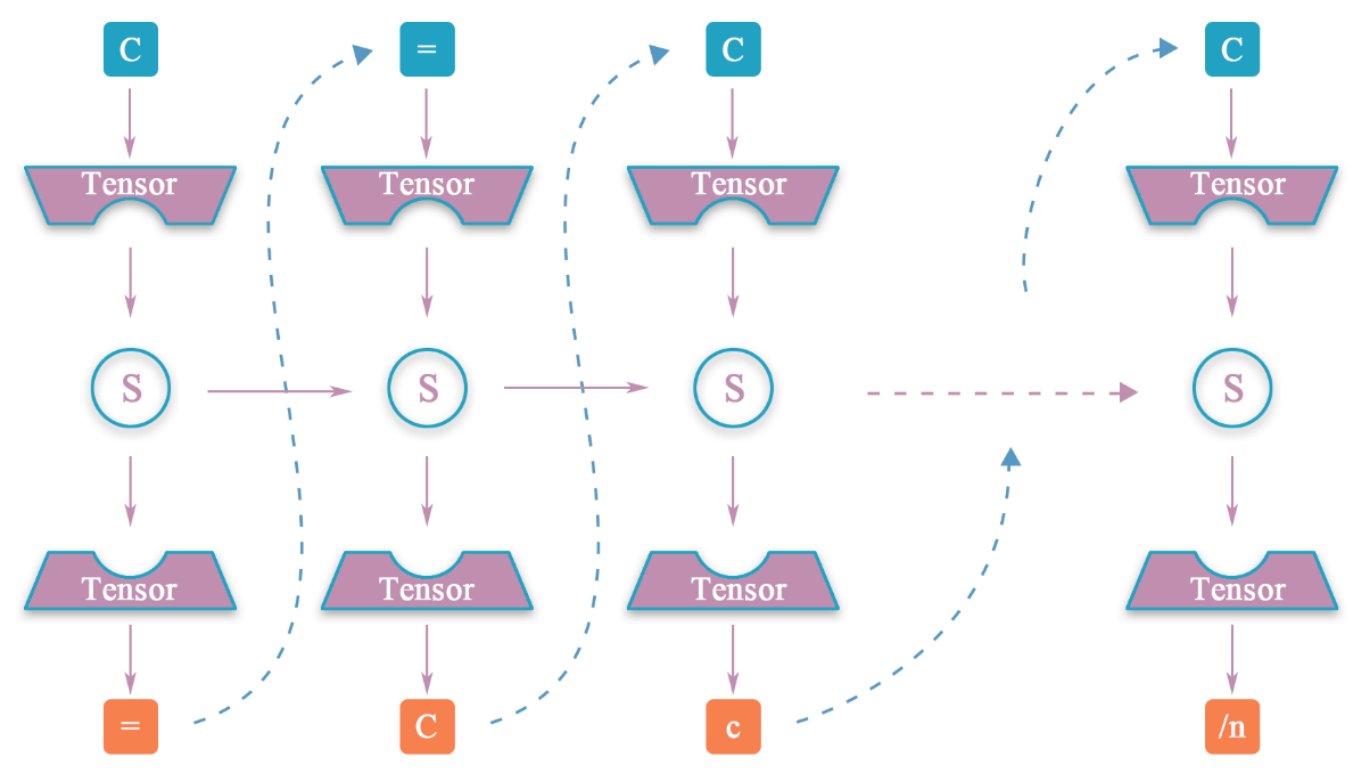

Figure 10. The general flow of reaction formation. We randomly selected a symbol $\mathrm{C}$ to start the generation, a tensor of each character of vocab then imports into the model and the probability distribution $\mathrm{P}$ as an outcome. According to the distribution $\mathrm{P}$, the model randomly selected the next symbol. Above steps will be cyclically execute until the appearance of EOF. Finally, the SMILES string as the result to output.

9, we apply c1ccccc1 to present benzene, character ">>" to separate reactants and products, "." to separate different reactants. Before the training step, the model will construct a vocab $(v 1, \ldots$, vi) that contains all characters in the SMILES strings.

Our inferenced part learns from the inference of poems (https://github.com/GaoPeng97/transformer-xl-chinese.git). This part inference the whole reaction with a start symbol. The model gives a tensor $\mathrm{T}(\mathrm{t} 1, \ldots, \mathrm{t})$ based on the start symbol and the data from the training step. The model then outcome the probability distribution $P(s 1, \ldots, s i)$ of the next symbol. The distribution $P$ is estimated based on the tensor $T$ and the built-in functions softmax in the tensorflow, which is defined as

$$
P\left(s_{k}\right)=\frac{\exp \left(t_{k}\right)}{\sum_{k^{\prime}=1}^{i} \exp \left(t_{k^{\prime}}\right)}
$$

Where tk corresponds to the kth element of tensor $\mathrm{T}$. Then the model randomly selects the next symbol according to the probability $\mathrm{P}$, and feedback to the model to find the following symbol (see Figure 10). To indicate the SMILES string happens to be a reaction, we lead the character $¥ n$ into each reaction SMILES as "end of line" (EOF). So the model will outcome the result and restart the generation of SMILES string from scratch when it detects the formation of EOF.

\section{Acknowledgements}

This project was supported by the National Natural Science Foundation of China, NSFC (Grant No.81903438).

\section{Conflict of interest}

The authors declare no conflict of interest.

Keywords: Deep learning $\cdot$ Heck reactions $\bullet$ Reaction generation $\cdot$ Transformer-XL

[1] J. Meisenheimer, Ber. 1919, 52B, 1929-1933.

[2] R. F. Kleinschmidt, A. C. Cope, J. Am. Chem. Soc. 1944, 66, 1929-1933.

[3] M. H. Todd, Chem. Soc. Rev. 2005, 34, 247-266.

[4] A. Cook, A. P. Johnson, J. Law, M. Mirzazadeh, O. Ravitz, A. Simon, Wiley Interdiscip. Rev. Comput. Mol. Sci. 2012, 2, 79-107.

[5] W. Beker, E. P. Gajewska, T. Badowski, B. A. Grzybowski, Angew. Chem. Int. Ed. 2019, 58, 4515-4519.

[6] T. J. Struble, J. C. Alvarez, S. P. Brown, M. Chytil, J. Cisar, R. L. DesJarlais, O. Engkvist, S. A. Frank, D. R. Greve, D. J. Griffin, X. Hou J. W. Johannes, C. Kreatsoulas, B. Lahue, M. Mathea, G. Mogk, C. A Nicolaou, A. D. Palmer, D. J. Price, R. I. Robinson, S. Salentin, L. Xing T. Jaakkola, W. H. Green, R. Barzilay, C. W. Coley, K. F. Jensen, J. Med. Chem. 2020, 63, 8667-8682.

[7] Y. Zhang, L. Wang, X. Wang, C. Zhang, J. Ge, J. Tang, A. Su, H. Duan, Org. Chem. Front. 2021, 8, 1415-1423.

[8] Y. Wu, C. Zhang, L. Wang, H. Duan, Chem. Commun. 2021, 57, 41144117.

[9] J. Law, Z. Zsoldos, A. Simon, D. Reid, Y. Liu, S. Y. Khew, A. P. Johnson, S. Major, R. A. Wade, H. Y. Ando, J. Chem. Inf. Model. 2009, 49, 593602.

[10] C. W. Coley, W. H. Green, K. F. Jensen, J. Chem. Inf. Model. 2019, 59, 2529-2537.

[11] W. Jin, C. W. Coley, R. Barzilay, T. Jaakkola, In Advances in Neural Information Processing Systems 2017, 2607-2616.

[12] C. W. Coley, W. Jin, L. Rogers, T. F. Jamison, T. S. Jaakkola, W. H. Green, R. Barzilay, K. F. Jensen, Chem. Sci. 2019, 10, 370-377.

[13] J. Nam, J. Kim, arXiv preprint arXiv:.09529 2016. 
[14] P. Schwaller, T. Gaudin, D. Lanyi, C. Bekas, T. Laino, Chem. Sci. 2018 9, 6091-6098.

[15] P. Schwaller, T. Laino, T. Gaudin, P. Bolgar, C. A. Hunter, C. Bekas, A A. Lee, ACS Cent. Sci. 2019, 5, 1572-1583.

[16] B. Liu, B. Ramsundar, P. Kawthekar, J. Shi, J. Gomes, Q. Luu Nguyen, S. Ho, J. Sloane, P. Wender, V. Pande, ACS Cent. Sci. 2017, 3, 1103 1113.

[17] G. Schneider, U. Fechner, Nat Rev Drug Discov 2005, 4, 649-663.

[18] M. Hartenfeller, G. Schneider, Wiley Interdiscip. Rev. Comput. Mol. Sci. 2011, 1, 742-759.

[19] Y. Xu, K. Lin, S. Wang, L. Wang, C. Cai, C. Song, L. Lai, J. Pei, Future Med. Chem. 2019, 11, 567-597.

[20] D. C. Elton, Z. Boukouvalas, M. D. Fuge, P. W. Chung, Mol. Syst. Des. Eng. 2019, 4, 828-849.

[21] Z. Wang, W. He, H. Wu, H. Wu, W. Li, H. Wang, E. Chen, arXiv preprint arXiv:.09889 2016

[22] M. H. S. Segler, T. Kogej, C. Tyrchan, M. P. Waller, ACS Cent. Sci. 2018 4, 120-131.

[23] H. Kim, J. Na, W. B. Lee, arXiv preprint arXiv:.00213 2021

[24] W. Bort, I. I. Baskin, T. Gimadiev, A. Mukanov, R. Nugmanov, P. Sidorov, G. Marcou, D. Horvath, O. Klimchuk, T. Madzhidov, Sci. Rep. 2021, 11 1-15.

[25] A. Vaswani, N. Shazeer, N. Parmar, J. Uszkoreit, L. Jones, A. N. Gomez, Ł. Kaiser, I. Polosukhin, in Advances in Neural Information Processing Systems, 2017, 5998-6008

[26] C. Zhang, X. Cai, H. Qiao, Y. Zhang, Y. Wu, X. Wang, H. Xie, F. Luo, H. Duan, ChemRxiv preprint ChemRxiv: 10.33774/chemrxiv-2021-fxvwg 2021.

[27] A. C. Vaucher, P. Schwaller, J. Geluykens, V. H. Nair, A. Iuliano, T. Laino, Nat. Commun. 2021, 12, 2573.

[28] Z. Dai, Z. Yang, Y. Yang, J. Carbonell, Q. V. Le, R. Salakhutdinov, arXiv preprint arXiv:.02860 2019.

[29] R. F. Heck, J. Am. Chem. Soc. 1968, 90, 5518-5526.

[30] L. Van der Maaten, G. Hinton, J. Mach. Learn.Research 2008, 9, 2579 2605.

[31] G. Hinton, S. T. Roweis, in NIPS, Vol. 15, Citeseer, 2002, 833-840.

[32] P. Schwaller, D. Probst, A. C. Vaucher, V. H. Nair, D. Kreutter, T. Laino, J.-L. Reymond, Nat. Mach. Intell. 2021, 3, 144-152.

[33] D. Probst, J. L. Reymond, J Cheminform 2020, 12, 12.

[34] X. Cheng, Z. Chen, Y. Gao, F. Xue, C. Jiang, Org. Biomol. Chem. 2016 14, 3298-3306

[35] R. Grigg, V. Sridharan, P. Stevenson, T. Worakun, J. Chem. Soc., Chem Commun. 1986, 1697-1699. 